\title{
COEFFICIENTS THERMIQUES ET ẼCOLOGIE DE QUELQUeS PLANAIRES D'EAU DOUCE
}

\author{
6. - DUGESIA TIGRINA
}

\author{
par R. RusSiER-Delolme.
}

La présence de Dugesia tigrina, «immigrant américain 》 est signalée dans la région lyonnaise en 1970 par Russier et LAScombe. Cette Planaire, à reproduction asexuée, occupe des eaux stagnantes dont la température atteint des valeurs estivales moyennes assez élcvées (voisines de $20^{\circ} \mathrm{C}$ ) et peut être fluctuante.

Les exemplaires étudiés ici proviennent de l'étang des Bruyères situé près du ruisseau de Méginand et décrit par Russien et Lasсомве $(1970)$. Ils peuvent être récoltés en grand nombre dans la partie aval de l'élang alors que Polycelis felina est présente à l'arrivée des sources et que $P$. nigra occupe la zone intermédiaire.

\section{I. - TOLÊRANCE THERMIQUE}

Différents auteurs indiquent la limite thermique supérieure de D. tigrina, Аввотт (1960) situe sa plus haute température de survie respectivement à $33,7^{\circ} \mathrm{C}$ ou $37,7^{\circ} \mathrm{C}$ sclon que l'expérience est faite à température constante, ou à température croissante. en augmentant celle-ci de $0,2^{\circ} \mathrm{C}$ toutes les $5 \mathrm{mn}$ à partir de $20^{\circ} \mathrm{C}$. Chanderer (1965) étudiant les capacités d'acclimatation de cette Planaire obtient après 2 semaines $100 \%$ de survie à $33^{\circ} \mathrm{C}$ et $65 \%$ à $35{ }^{\circ} \mathrm{C}$. Daнm (1958) qualifie $D$. tigrina d'eurytherme car elle vil dans des eaux dont la température est comprise entre $9^{\circ}$ et $25^{\circ} \mathrm{C}$. Il la récolte même dans des aquariums de serres chaudes en Angleterre, au Danemark, en Suède.

La publication de Russier et Lascombe, citée précédemment, a montré que les populations de $D$. tigrina acclimatées dans la région lyonnaise sont plus tolérantes à la chalcur que les espèces indigènes : leur temps de survie aux températures élevées est toujours

1. Equipe d'Ecophysiologie, Biologie Animale, Université Claude-Bernard Lyon I, boulevard du 11-Novembre, 69-Villeurbanne. 
supérieur à celui de ces dernières. Grâce à un complément de données, l'étude statistique a pu être réalisée récemment et l'incertitude quant à la linéarité du tracé présenté en 1970 sera donc levée. Cette étude porte sur les temps individuels de survie, exprimés en logarithmes, pour des animaux récoltés en hiver d'une part, en été d'autre part.

La transformation logarithmique (calcul de la moyenne géométrique des temps individuels de survie) recommandée par Fry, Hart et Walker (1946) et utilisée par PatTeE (1966 et 1968) réduit considérablement la variabilité individuelle. C'est ce que montre le calcul de l'écart-type des moyennes au tableau I (première partie).

\section{A. - D. tigrina récoltées en hiver.}

Les animaux ont été récoltés le 27-12-1968 (température de l'eau : $4,5^{\circ} \mathrm{C}$ ) et acclimatés au laboratoire à $10^{\circ} \mathrm{C}$ pendant 12 jours.

Le test de linéarité portant sur l'ensemble des résultats obtenus aux 4 températures : $30^{\circ}, 32,5^{\circ}, 35^{\circ}, 37,5^{\circ}$ (tableau I A) indique que les termes non linéaires sont hautement significatifs. Il n'est donc pas possible de représenter la survie moyenne en fonction de la température par une droite unique.

Si l'on considère seulement les temps de survie aux températures $\geqslant 32,5^{\circ} \mathrm{C}$ (tableau I B) les termes non linéaires ne sont pas significatifs : la portion de tracé correspondant à ces 3 températures doit être considérée comme rectiligne. Il existe donc un point d'inflexion à $32,5^{\circ} \mathrm{C}$. Nous pouvons représenter les variations du temps de survie en fonction de la température par deux segments rectilignes (fig. 1 ).

Pour compléter ces résultats, il est utile de déterminer les pentes des deux segments et leurs intervalles de confiance au seuil de $5 \%$ en utilisant le test $t$ de Student. Ces valeurs sont les suivantes :

$-0,37$ (-0,33 à $-0,40)$ pour le segment correspondant aux températures $\geqslant 32,5^{\circ} \mathrm{C}$.

$-0,06(-0,16$ à $+0,05)$ pour le segment correspondant aux lempératures $\leqslant 32,5^{\circ} \mathrm{C}$.

La différence entre les 2 coefficients de régression est donc significative. La pente de la droite obtenue pour les températures $\leqslant 32,5^{\circ} \mathrm{C}$ n'est pas significativement différente de zéro. On ne peut pas dire que cette droite s'écarte de l'horizontale. 
Tableau I. - Durée moyenne de survie de D. tigrina à température constante. Début des mesures : 27 décembre 1968.

Première partie du tablean : moyenne des temps individuels de survie. Les temps sont exprimés en jour \pm l'écart-type. $N=$ nombre d'animaux en expérience.

Deuxième partie du tableau : analyse de variance des données logarithmiques pour :

A) 4 températures : $37,5^{\circ} \mathrm{C}-35^{\circ} \mathrm{C}-32,5^{\circ} \mathrm{C}-30^{\circ} \mathrm{C}$

B) 3 températures : $37,5^{\circ} \mathrm{C}-35^{\circ} \mathrm{C}-32,5{ }^{\circ} \mathrm{C}$.

La colonne seuils représente les valeurs critiques de la distribution de $F$, pour le nombre de degrés de liberté (D. L.) indiqué et les niveaux de probabilité de $5 \%$ et $1 \%$.

$\mathrm{F}^{\star \star}$ : la valeur de $\mathrm{F}$ dépasse le niveau de $1 \%$ (différence hautement significative).

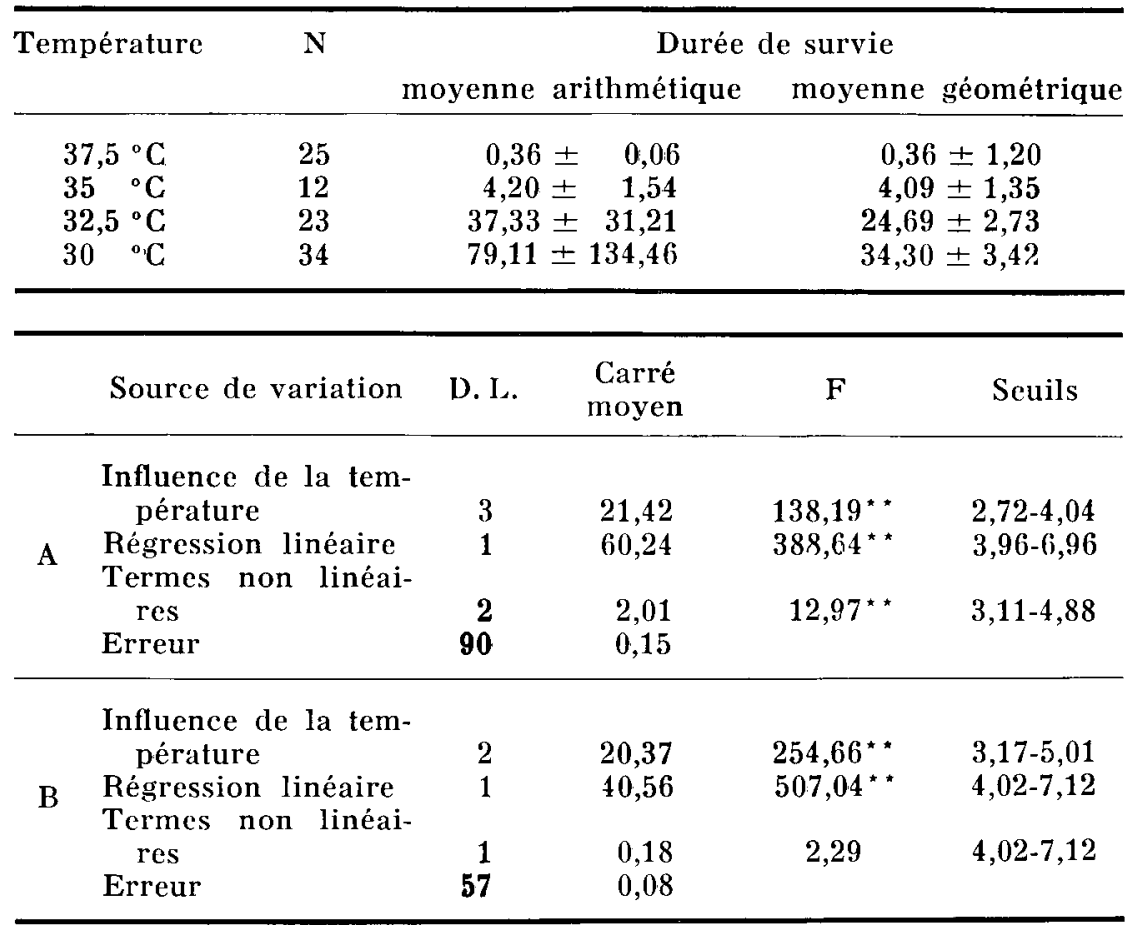

\section{B. - D. tigrina récoltées en été.}

La température de l'eau à l'étang le 25-9-1969, au moment de la récolte étant de $10^{\circ} \mathrm{C}$, le réchauffement graduel des animaux jusqu'aux températures d'expérience a commencé, ce même jour, à $20^{\circ} \mathrm{C}$.

Les résultats sont rasscmblés au tableau II. Comme pour les animaux récoltés en hiver, l'analyse statistique indique que la durée de survie varie en fonction de la température et qu'il existe 
un point d'inflexion à $32,5^{\circ} \mathrm{C}$. Les variations du temps moyen de survie en fonction de la température sont représentées par 2 segments de droite (fig. 1). Les pentes de ces segments et leurs intervalles de confiance au seuil de probabilité de $5 \%$ sont :

$-0,28(-0,26$ à $-0,31)$ pour les températures $\geqslant 32,5^{\circ} \mathrm{C}$.

$-0,04(-0,03$ à $-0,12)$ pour les températures $\leqslant 32,5^{\circ} \mathrm{C}$.

Tableau II. - Durée moyenne de survie de $D$. tigrina à température constante. Début des mesures : 25 septembre 1969.

Même légende qu'au tableau I.

Première partic du tableau : moyenne des temps individuels de survie.

Deuxième partie du tableau : analyse de variance des données logarithmiques.

A) pour les 4 températures

B) pour les 3 températures les plus élevées.

\begin{tabular}{|c|c|c|c|c|c|c|}
\hline \multicolumn{2}{|c|}{ Température } & $\mathbf{N}$ & \multicolumn{4}{|c|}{$\begin{array}{l}\text { Durée de survie } \\
\text { moyenne arithmétique moyenne géométrique }\end{array}$} \\
\hline \multicolumn{2}{|c|}{$\begin{array}{l}37,5^{\circ} \mathrm{C} \\
35{ }^{\circ} \mathrm{C} \\
32,5^{\circ} \mathrm{C} \\
30{ }^{\circ} \mathrm{C}\end{array}$} & $\begin{array}{l}40 \\
39 \\
49 \\
61\end{array}$ & \multicolumn{2}{|c|}{$\begin{array}{r}1,00 \pm 0,07 \\
4,50 \pm 1,76 \\
34,43 \pm 19,68 \\
68,04 \pm 72,56\end{array}$} & \multicolumn{2}{|c|}{$\begin{array}{r}0,99 \pm 1,07 \\
3,99 \pm 1,81 \\
25,95 \pm 2,37 \\
33,33 \pm 3,70\end{array}$} \\
\hline \multicolumn{3}{|c|}{ Source de variation } & D. I. & $\begin{array}{l}\text { Carré } \\
\text { moyen }\end{array}$ & $\mathbf{F}$ & Seuils \\
\hline \multicolumn{3}{|c|}{$\begin{array}{l}\text { Influence de la tem- } \\
\text { pérature } \\
\text { Régression linéaire } \\
\text { Termes non linéai- } \\
\quad \text { res } \\
\text { Erreur }\end{array}$} & $\begin{array}{r}3 \\
1 \\
2 \\
185\end{array}$ & $\begin{array}{l}23,83 \\
66,23\end{array}$ & $\begin{array}{c}149,22^{* *} \\
441,53^{* *} \\
17,53^{* *}\end{array}$ & $\begin{array}{l}2,67-3,91 \\
3,91-6,81 \\
\\
3,06-4,75\end{array}$ \\
\hline B & $\begin{array}{l}\text { Influenc } \\
\text { pératy } \\
\text { Régressi } \\
\text { Termes } \\
\quad \text { res } \\
\text { Erreur }\end{array}$ & $\begin{array}{l}\text { ure de la tem- } \\
\text { ion linéaire } \\
\text { non linéai- }\end{array}$ & $\begin{array}{r}1 \\
125\end{array}$ & $\begin{array}{l}22,55 \\
44,81\end{array}$ & $\begin{array}{l}304,73^{\star *} \\
605,54^{\star *}\end{array}$ & $\begin{array}{l}3,07-4,78 \\
3,92-6,84\end{array}$ \\
\hline
\end{tabular}

C. - Influence de la saison de récolte sur la durée de survie aux températures élevéos.

1) Températures $\geqslant 32,5^{\circ} \mathrm{C}$.

Le test est effectué par l'analyse de covariance et par comparaison des intervalles de confiance des deux coefficients de régression. 


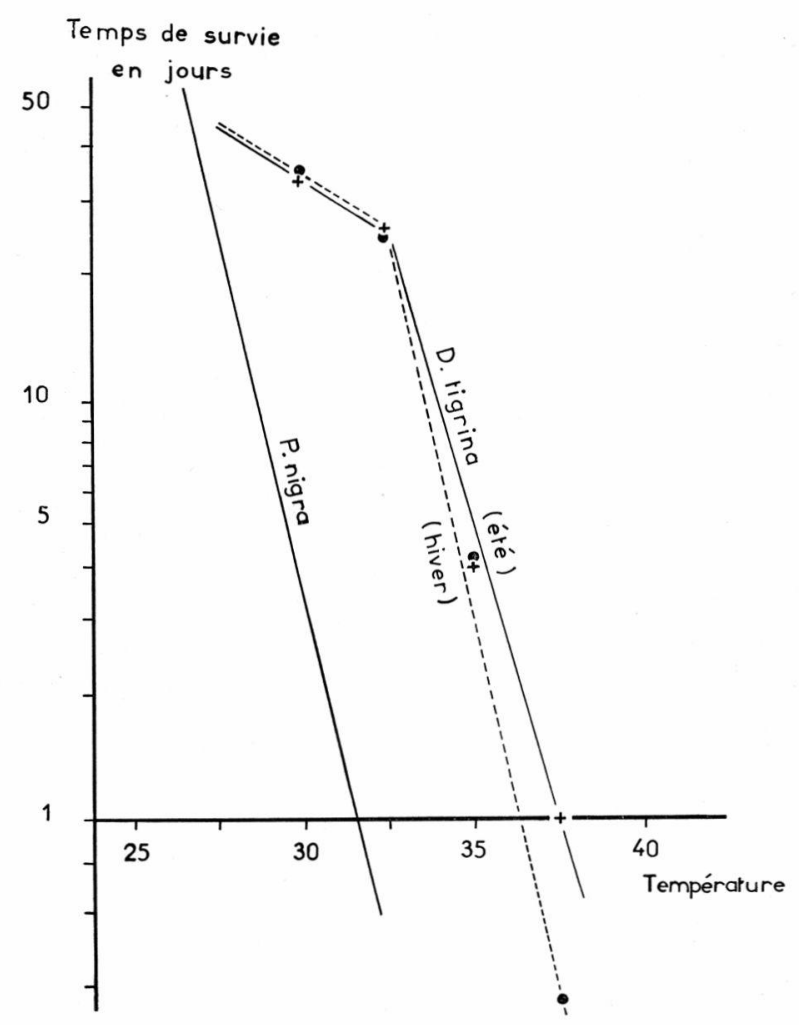

Fig. 1. - Influence de la température sur la durée moyenne de survie de Dugesia tigrina et Polycelis nigra.

La droite de $P$. nigra est transcrite de la publication de Pattee (1966). Pour D. tigrina, pente des segments de droite et limites de l'intervalle de confiance au seuil de probabilité de $5 \%$ :

- Températures $\leqslant 32,5^{\circ} \mathrm{C}$ :

Pente commune aux deux segments : $-0,05(-0,11$ à $+0,01)$

- Températures $\geqslant 32,5^{\circ} \mathrm{C}$ :

Eté : $0,28(-0,26$ à $-0,31)$

Hiver : $-0,37(-0,33$ à $-0,40)$.

L'analyse de covariance porte sur l'ensemble des données exprimées en logarithmes et obtenues, en hiver et en été, aux températures $\geqslant 32,5^{\circ} \mathrm{C}$. Elle revient à comparer les durées de survie comme si elles avaient été mesurées à une même température et est effectuée selon la méthode des Y réduits (Lison 1958). Elle compare donc les points centraux des deux droites de régression et indique la position de l'une par rapport à l'autre.

Le tableau III A résume cette analyse. La différence entre les durées de survie en été et en hiver est hautement significative. Le 
segment de droite «été » est, par rapport à celui d' «hiver», décalé vers les températures supérieures. Les individus récoltés à la fin de l'été, donc ayant subi une acclimatation à des températures élevées, résistent plus longtemps que les individus récoltés en hiver. Pour les températures indiquées, l'effet de l'acclimatation thermique naturelle est identique à celui de l'acclimatation au laboratoire come l'a montré MATH!EU (1971) pour Niphargus longicaudatus.

Les intervalles de confiance des pentes des deux segments de droite sont indiqués aux paragraphes $A$ et $B$. On voit que les pentes diffèrent de façon significative : les deux segments de droite convergent vers le haut.

Tableau III. - Influence de la saison de récolte sur la durée de survie.

Analyse de covariance, effectuée selon la méthode des Y réduits (LisoN 1958), pour l'ensemble des données exprimées en logarithmes et obtenues à :

A) 3 températures élevées : $37,5^{\circ} \mathrm{C}-35^{\circ} \mathrm{C}-32,5^{\circ} \mathrm{C}$

B) 2 températures plus basses : $32,5^{\circ} \mathrm{C}-30^{\circ} \mathrm{C}$.

Les valeurs critiques de $\mathbf{F}$, pour le nombre de degrés de liberté (D. L.) et les niveaux de probabilité de $5 \%$ et $1 \%$ sont indiquées dans la colonne seuils.

\begin{tabular}{|c|c|c|c|c|c|}
\hline & Source de variation & D. L. & $\begin{array}{c}\text { Carré } \\
\text { moyen }\end{array}$ & $\mathrm{F}$ & Seuils \\
\hline A & $\begin{array}{l}\text { Influence de la sai- } \\
\quad \text { son } \\
\text { Erreur }\end{array}$ & $\begin{array}{r}1 \\
185\end{array}$ & $\begin{array}{l}1,19406 \\
0,06641\end{array}$ & $17,98^{\star *}$ & $3,91-6,81$ \\
\hline B & $\begin{array}{l}\text { Influence de la sai- } \\
\text { son } \\
\text { Erreur }\end{array}$ & $\begin{array}{r}1 \\
164\end{array}$ & $\begin{array}{l}0,58337 \\
0,24239\end{array}$ & 2,41 & $3,91-6,81$ \\
\hline
\end{tabular}

2) Températures $\leqslant 32,5^{\circ} \mathrm{C}$.

A $32,5^{\circ} \mathrm{C}$ et $30^{\circ} \mathrm{C}$, la saison n'a aucune influence sur la durée moyenne de survie (tableau III B). Les deux segments de droite sont situés au même niveau. La différence entre les deux coefficients de régression n'étant pas significative, on peut aussi considérer yu'ils ont la même pente. Nous pouvons donc représenter graphiquement les variations du temps moyen de survie en fonction de ces températures, par un segment de droite unique pour les expériences d'hiver et d'été. Cette droite, avec un coefficient de régression de $-0,05(-0,11$ à $+0,01)$, ne diffère pas significativement d'une horizontale. 


\section{D. - Discussion des tracés obtenus.}

Les courbes de Pattee (1966 et 1968) pour Polycelis felina, $P$. nigra, Crenobia alpina et Dugesia gonocephala avaient une allure rectiligne. Chez nous il existe manifestement une rupture de pente à $32,5^{\circ} \mathrm{C}$ qui correspond à un changement dans l'influence de la température sur la durée de survie. Nous avons vu qu'aux températures les plus élevées, $35^{\circ} \mathrm{C}$ et $37,5{ }^{\circ} \mathrm{C}$, les animaux meurent sans se multiplier. Par contre à $30{ }^{\circ} \mathrm{C}$ et $32,5^{\circ} \mathrm{C}$ la multiplication asexuée continue. La présence de cette multiplication à certaines températures et pas à d'autres, la résistance moindre des jeunes nés en cours d'expérience par rapport à celle des parents mis au départ sont donc probablement les causes de l'inflexion à $32,5^{\circ} \mathrm{C}$.

Seule la partie de la droite concernant les températures $\geqslant 32,5^{\circ} \mathrm{C}$ peut être comparée avec les courbes obtenues par PatTeE pour des individus récoltés en été qui ne se multiplient pas non plus aux températures testées. Ce segment de droite est parallèle aux tracés indiqués pour $P$. nigra et $P$. felina. Les pentes sont voisines $(P$. nigra : $-0,31, P$. felina : $-0,37)$. Pour $D$. tigrina le tracé est décalé de $5{ }^{\circ} \mathrm{C}$ vers les températures supérieures. Ce résultat est bien en accord avec la situation qu'elle occupe sur le terrain. Néanmoins les relevés des maximums de température à l'étang des Bruyères et l'étude de la tolérance des espèces indigènes nous amènent à penser que la cause réelle de la zonation des espèces dans la nature ne réside pas dans l'effet létal de la température mais plutôt dans l'influence de cette dernière sur les capacités physiologiques et en particulier la reproduction de l'animal. Nous avons étudié cette reproduction à différentes températures.

\section{II. - REPRODUCTION}

Les animaux qui ont servi de parents à tous les élevages ont été récoltés à l'étang des Bruyères en mars 1969. Tous présentent un mode de reproduction asexué par scission postpharyngienne.

Les élevages ont lieu en eau stagnante. La technique est identique à celle indiquée par Pattee (1969).

\section{A. - Influence de températures constantes sur le taux de repro- duction.}

1) Températures basses.

a) Température de $5^{\circ} \mathrm{C}$.

- Les adultes élevés dans un réfrigérateur vitré et nourris toutes les semaines ne se sont pas reproduits. Après un an d'éle- 
vage le taux de mortalité n'est que de $30 \%$, mais tous les individus sont inactifs et certains ont l'exprémité postérieure en voie de décomposition. Leur taille a diminué de moitié.

- Les fragments détachés à $15^{\circ}$ (température la plus basse à laquelle il soit possible d'en obtenir), ou à $23^{\circ}$ et élevés à $5^{\circ}$ ne régénèrent pas ou très mal. Une zone de tissu plus claire apparaît au niveau de la section mais le pharynx n'est pas régénéré. Les taches oculaires n'ont été visibles que dans 2 cas seulement sur les 60 fragments suivis, soit $3 \%$, au bout de 70 jours et 110 jours. La taille des fragments diminue jusqu'à $2 \mathrm{~mm}$, puis ils se décomposent. La survie moyenne des fragments est de $106 \pm 6$ jours.

\section{b) Température de $10{ }^{\circ} \mathrm{C}$.}

- Les adultes survivent bien à $10{ }^{\circ} \mathrm{C}$. Ils se nourrissent et atteignent des tailles considérables, jusqu'à $13 \mathrm{~mm}$ (moyenne : $10,3 \mathrm{~mm}$ ). Partant de 18 animaux que j'ai élevés plus d'un an, je n'ai obtenu aucune fragmentation spontanée. Par contre j'ai eu un grand nombre de fragmentations accidentelles, prarfois à la suite d'une blessure, mais plus fréquemment d'une panne du réf rigérateur.

Les élevages à $5^{\circ}$ et $10^{\circ}$ avaient lieu dans 2 compartiments différents de la même étuve. Au moment des pannes, la température atteignait $20^{\circ}$ dans chacun d'eux. A la suite de cette augmentation de température je n'ai constaté aucune coupure à $5^{\circ}$, par contre elles étaient nombreuses à $10^{\circ}$ même si l'élévation de la température ne durait qu'une nuit.

- Les 37 fragments détachés à $15^{\circ}$ ou $23^{\circ}$ et élevés à $10^{\circ}$ ont régénéré des adultes de grande taille. Ces derniers ne se sont pas fragmentés, sauf après une augmentation accidentelle de la température. Les 17 fragments caudaux obtenus ainsi en 21 mois régénèrent en 47 jours. C'est à $10^{\circ}$ que les animaux atteignent leur taille maximum mais le taux de reproduction naturelle étant nul, les lignées finissent nécessairement par disparaître au bout d'un temps long (plusieurs années).

Le résultat des élevages à des températures constantes de $5^{\circ}$ ou $10^{\circ}$ est donc le même. Ces 2 températures ne permettent pas le maintien de l'espèce car la fragmentation est inhibéc. A $10^{\circ}$ seulement, les animaux s'alimentent. L'augmentation de leur taille les met alors en état de se fragmenter dès qu'un réchauffement se produit. Ce même réchauffement n'a aucune action à $5^{\circ}$.

2) Température Élevée $: 27,5{ }^{\circ} \mathrm{C}$.

Une population de D. tigrina issue de 10 animaux entiers de taille importante a survécu environ 2 ans à $27,5^{\circ} \mathrm{C}$. A cette tem- 
pérature, les adultes se fragmentent (périodicité moyenne de fragmentation : 57,6 jours). Au cours de l'expérience nous assistons à une diminution progressive de la taille des parents. La fragmentation et le métabolisme élevé à cette température provoquent un déficit de matière vivante qui n'est pas entièrement compensé par l'absorption de nourriture. Les divisions sont de plus en plus rares et, parallèlement à ceci, la longueur des fragments est de plus en plus petite. Cette longueur est proportionnelle à la taille des parents. Au-dessous de certaines dimensions les fragments ne sont plus viables. Lorsque la deuxième génération se développe, les fragments qu'elle produit ne survivent jamais.

A $27,5{ }^{\circ} \mathrm{C}$ comme à $10^{\circ} \mathrm{C}$, la population disparaît. Dans le premier cas cela est dû, en partie, à la mortalité des fragments, dans le deuxième cas à l'absence de fragmentation.

3) Températures moyennes : $15^{\circ} \mathrm{C}$ à $25^{\circ} \mathrm{C}$.

En Amérique, Chandler (1965) obtient une multiplication asexuée entre $19{ }^{\circ} \mathrm{C}$ et 29 à $31{ }^{\circ} \mathrm{C}$ avec un maximum à $25^{\circ} \mathrm{C}$. Pour Kenk (1937), la reproduction commence à une température comprise entre $10{ }^{\circ} \mathrm{C}$ et $12{ }^{\circ} \mathrm{C}$ à condition que les animaux aient atteint une taille minimum.

Dans mes élevages, le détachement des fragments caudaux qui n'était pas possible à $10^{\circ}$ alors que leur régénération l'était, s'effectue à toutes les autres températures indiquées. L'intensité de la scissiparité, seul mode de reproduction, paraît liée à la température. Nous pouvons donc situer les limites de la zone dans laquelle D. tigrina se reproduit chez nous. Pour les températures inférieures, celte limite est entre $10^{\circ} \mathrm{C}$ et $15^{\circ} \mathrm{C}$, pour les températures supérieures elle est entre $25^{\circ} \mathrm{C}$ el $27,5{ }^{\circ} \mathrm{C}$. Les résultats figurent au tableau IV. Il donne la valeur, pour $D$. tigrina, des mêmes paramètres que ceux mesurés par PatTee (1969 et 1970) pour les Planaires indigènes.

Le nombre d'individus étudiés à chaque température (ligne 1) est le nombre de «parents» mis au départ, c'est-à-dire de fragments détachés à la température d'expérience, ou à $23^{\circ}$ pour les animaux élevés à $15^{\circ}$ car la multiplication est trop lente à cette dernière température.

La ligne 2 du tableau montre que le détachement des fragments s'accélère à partir de $15^{\circ}$ pour atteindre son maximum à $25^{\circ}$. Entre ces 2 températures le taux de mortalité des fragments (ligne 3) varie peu. La périodicité moyenne de production d'un jeune viable (ligne 4) et la durée d'une génération (ligne 6) sont calculées en faisant la moyenne des durées pour les différents 


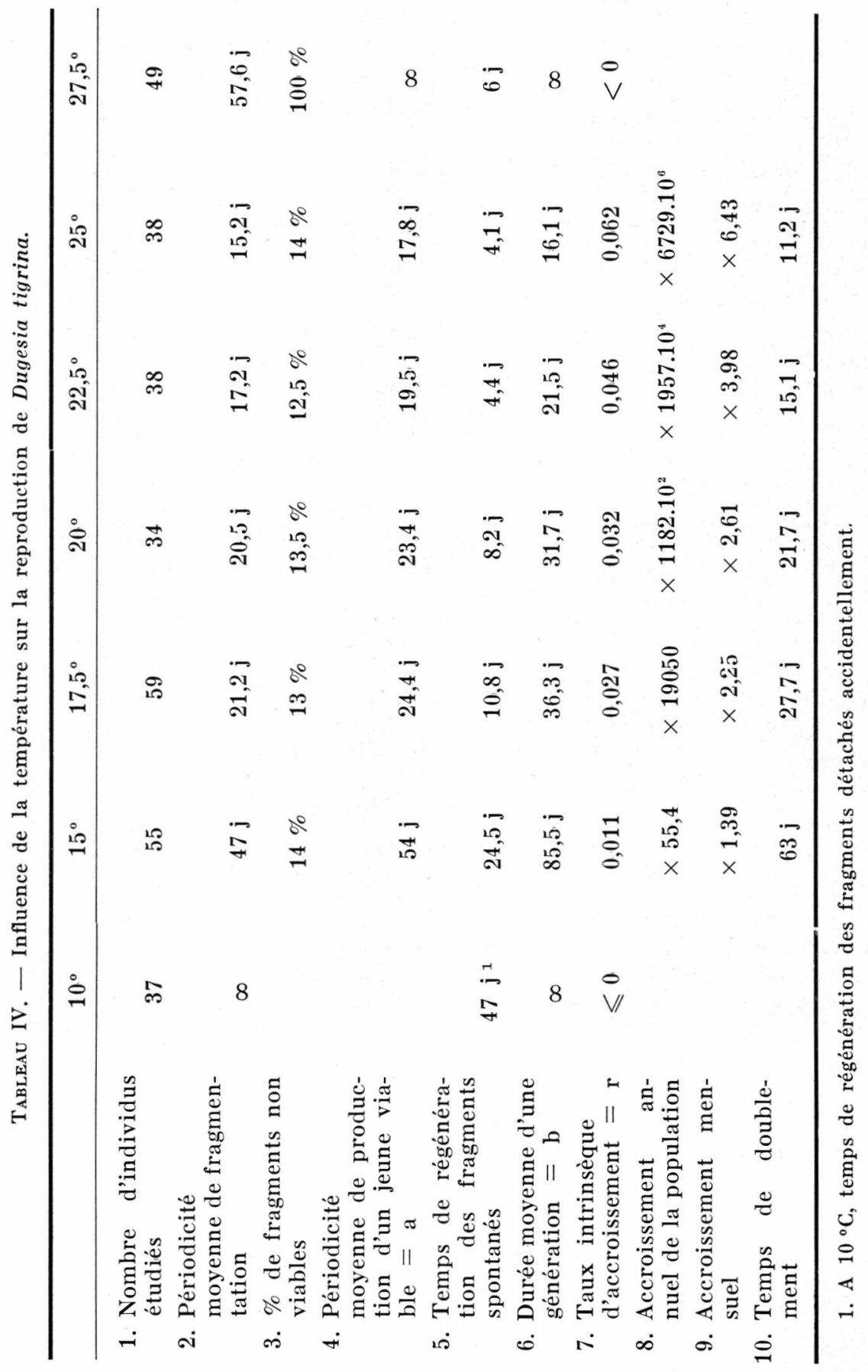


individus. Elles ont leur valeur minimum à $25^{\circ} \mathrm{C}$, d'où le maximum du taux intrinsèque d'accroissement à cette température (ligne 7 ).

L'influence de la température sur les capacités de multiplication apparaît de façon encore plus précise si l'on considère la suite du tableau.

Lorsque l'effectif initial $N_{0}$ d'une population est connu, il est possible de calculer, au temps $t$, le nombre $\mathbf{N}_{t}$ d'individus qu'atteindrait cette population dans un milieu où l'espace et la nourriture seraient en quantités illimitées. Il suffit d'appliquer la formule :

$$
\begin{aligned}
& N_{t}=N_{0} e^{r t} \\
& \text { avec } N_{t}=\text { effectif au temps } t \\
& \mathbf{N}_{\mathrm{o}}=\text { effectif initial } \\
& \mathrm{e}=\text { base des logarithmes népériens } \\
& \mathbf{r}=\text { taux intrinsèque d'accroissement naturel de la popu- }
\end{aligned}
$$

Pour fixer les idées, est porté aux lignes 8 et 9 le nombre théorique de descendants ainsi produits par un individu. Ce nombre est calculé respectivement après 1 an et après 1 mois d'élevage à la température considérée. Le chiffre indiqué représente le coefficient par lequel le nombre d'animaux serait multiplié chaque année ou chaque mois. De la même façon, la formule précédente permet de calculer le nombre de jours nécessaire au doublement de la population. Ce nombre est indiqué à la ligne 10 . DAнм (1958), étudiant l'influence de la nourriture des animaux sur la multiplication asexuée, note qu'une population nourrie tous les 10 jours voit, à une température variant entre $18{ }^{\circ} \mathrm{C}$ et $21{ }^{\circ} \mathrm{C}$, ses effectifs doubler en 5 semaines. Dans les conditions de mon élevage, la multiplication est plus rapide puisque le temps de doublement serait de 3 semaines.

\section{4) Conséquence : la taille des individus.}

Dans la littérature, de nombreuses données indiquent que la taille des individus est souvent fonction de la température.

VANDEL (1922), étudiant le processus de scission chez 4 espèces de Planaires, constate que les animaux qui se coupent activement sont en général de très petite taille. "Les cultures d'été où les scissions sont très fréquentes et les individus de petite dimension, contrastent fortement avec les cultures d'hiver, où les divisions sont rares et les individus de grande taille.» 
Abeloos (1929 et 1930) semble être le premier à donner des chiffres chez $D$. gonocephala. Le poids maximum de cette Planaire, pour des conditions de nutrition déterminées, est plus élevé à basse température. A $7^{\circ}$, il est presque triple de ce qu'il est à $20^{\circ}$.

Dans tous les élevages de $D$. tigrina effectués par DAнM (1955), la taille des individus diminue quand la température augmente.

KENK (1937) remarque qu'aux basses températures $D$. tigrina ne se divise pas avant d'atteindre une longueur considérable. La multiplication ne commence, à une température comprise entre $10^{\circ}$ et $12^{\circ}$, que si les animaux ont une taille minimum. Si la température est plus élevée, les coupures ont lieu plus tôt chez des animaux plus petits.

Après un séjour de plusieurs mois à température constante $\left(10^{\circ}\right.$, $15^{\circ}, 17,5^{\circ}, \ldots, 25^{\circ}$ ) les adultes, ayant jeûné une semaine, sont pesés et mesurés. Les surfaces externes étant séchées au moyen de papier filtre, le poids indiqué est le poids frais. La longueur est déterminée en plaçant les animaux dans une boîte de pétri contenant de l'eau et posée sur une feuille de papier millimétré. Les animaux sont mesurés quand ils ont une forme allongée, c'est-à-dire quand ils se déplacent dans la boîte. Une quinzaine de mesures de poids et de longueur ont été effectuées à chaque température; les résultats sont rassemblés au tableau V. L'analyse statistique, pratiquée sur l'ensemble des données, nous indique que la température a une influence hautement significative sur le poids et la longueur : la variation due à ce facteur n'est pas explicable par l'ampleur de la variation due à l'erreur. La figure 2, où sont représentées en fonction de la température les variations du poids, de la longueur et du taux intrinsèque d'accroissement de $D$. tigrina, permet de constater la relation entre la taille et l'intensité de la reproduction. Les échelles étant différentes, nous pouvons seulement comparer l'allure générale des tracés et non leur niveau ou leur pente.

A $5^{\circ}$ et $27,5^{\circ}$ les fragments mis en expérience ne se reproduisent pas ou donnent naissance à des jeunes non viables, $\mathbf{r}$ est $<0$. Le poids et la longueur d'animaux en équilibre à ces températures sont nuls. Si l'on excepte ces 2 températures extrêmes, on constate que la taille des individus, après un maximum à $10^{\circ}$, diminue quand la température augmente et ceci dès que la multiplication asexuéc commence, entre $10^{\circ}$ et $15^{\circ}$.

A $25^{\circ}$, au taux de multiplication maximum, correspondent des poids et longueurs minimums. A chaque température, il existe donc bien, pour les animaux, une taille d'équilibre. 


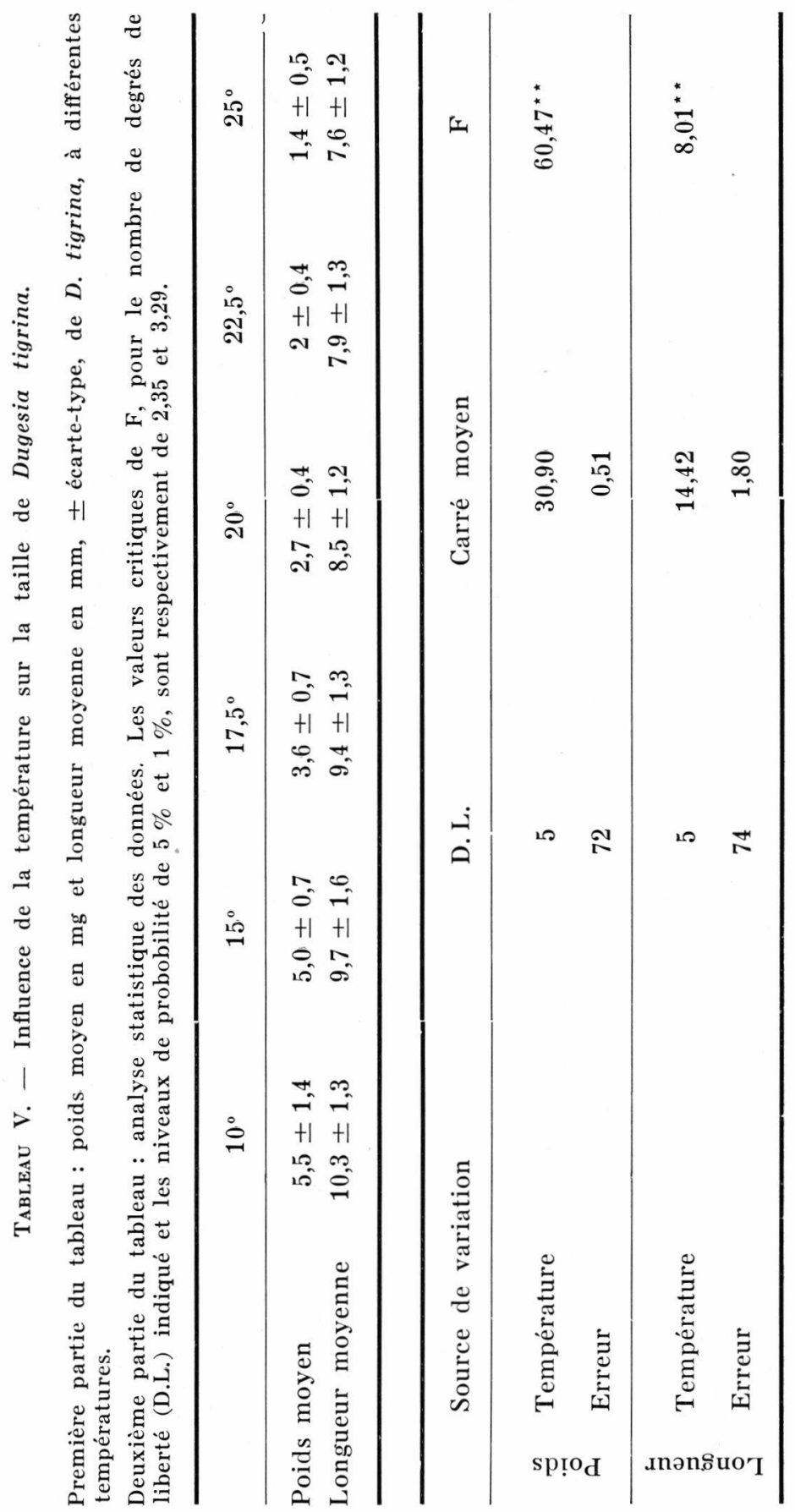




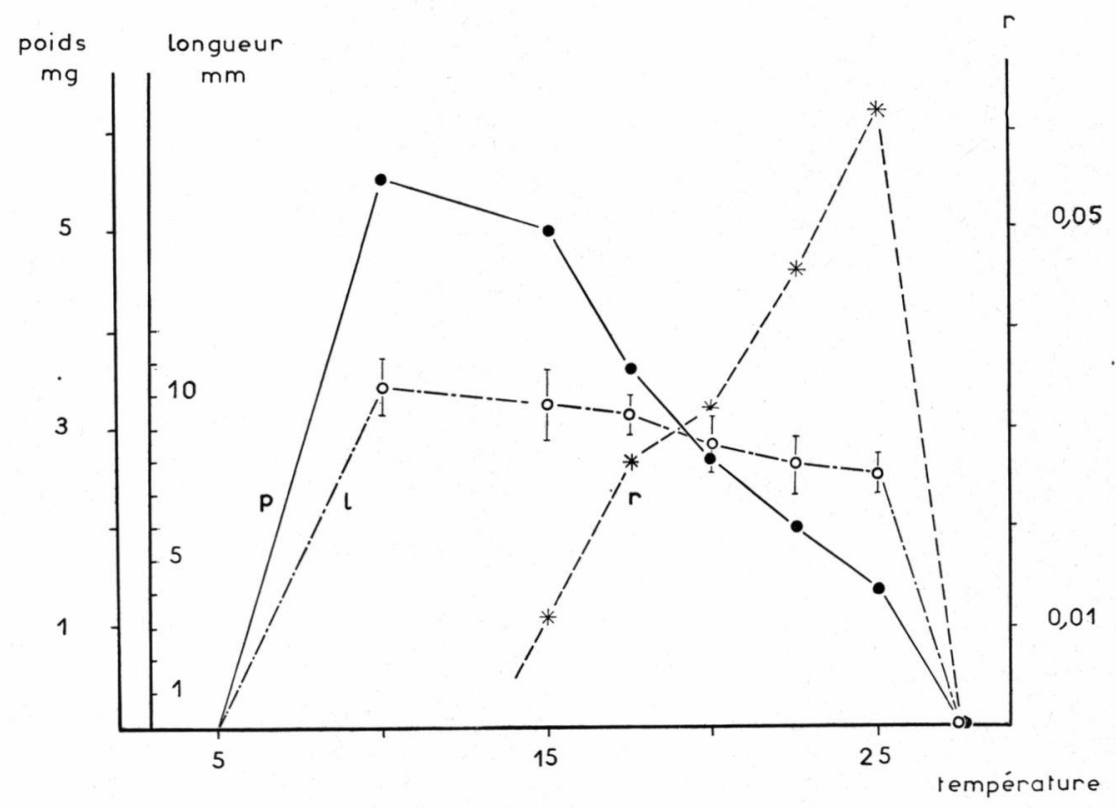

Fig. 2. - Variation du poids moyen (courbe p), de la longueur moyenne (courbe 1) et du taux intrinsèque d'accroissement (courbe $r$ ) en fonction de la température chez Dugesia tigrina. Les intervalles de confiance de la moyenne sont tracés pour la longueur.

\section{B. - Comparaison avec les Dugesia tigrina américaines et les espèces indigènes.}

1) Comparaison avec les populations américaines de Dugesia TIGRINA.

Seuls Kenk (1937) et Root (1960) ont calculé les paramètres de la reproduction de $D$. tigrina.

KENK mesure la périodicité de fragmentation de la race asexuée. Elle est égale à 1427 jours pour une température comprise entre $10^{\circ}$ et $12^{\circ}$, et à 15,8 jours pour une température comprise entre $20^{\circ}$ et $30^{\circ}$. Bien qu'il soit difficile d'établir des comparaisons, puisque la température n'est pas constante, il semble que dans les expériences de cet auteur la multiplication soit plus rapide que dans les nôtres où à $25^{\circ}$ la périodicité de production d'un jeune viable est de 17,8 jours.

Root recherche le taux intrinsèque d'accroissement naturel d'une population asexuée de $D$. tigrina en construisant la table de vie à $23^{\circ}$. Pour cela, il élève 26 animaux pendant des intervalles de temps variant entre 44 et 107 jours. Chaque animal est isolé dans un bol et nourri de Daphnies tous les 3 jours. En faisant la 
moyenne des 26 valeurs de $\mathrm{r}$ qui varient entre 0,051 et 0,091 , il obtient un taux intrinsèque moyen de 0,072 . Ce chiffre correspond, d'après CoLE (1960), à une périodicité moyenne de fragmentation de 6 jours et à une durée moyenne de génération de 15 jours. Le taux r, mesuré ici, n'atteint jamais la valeur de 0,072 . La différence tient peut-être en partie aux conditions expérimentales plus favorables dans l'expérience de Rоoт (1960). En effet, les individus étant isolés, il n'y a pas de cannibalisme. La nourriture est différente et plus abondante que dans notre expérience. Mais il semble, malgré tout, que $D$. tigrina dans la région lyonnaise soit moins prolifique que les populations américaines.

De plus en Amérique, $D$. tigrina se multiplie asexuellement jusqu'à $30^{\circ}$ ou $31^{\circ}$ (Chandler 1965, KEnK 1937). Mais ces auteurs ne précisent pas si les fragments vont viables. Les clones étudiés ici semblent moins thermophiles.

\section{2) Comparaison avec les espèces indigènes.}

La figure 3 permet de comparer les valeurs du taux intrinsèque d'accroissement pour 5 Planaires. Les espèces indigènes suivantes ont été étudiées par Pattee : C. alpina, P. felina, D. gonocephala et $P$. nigra.

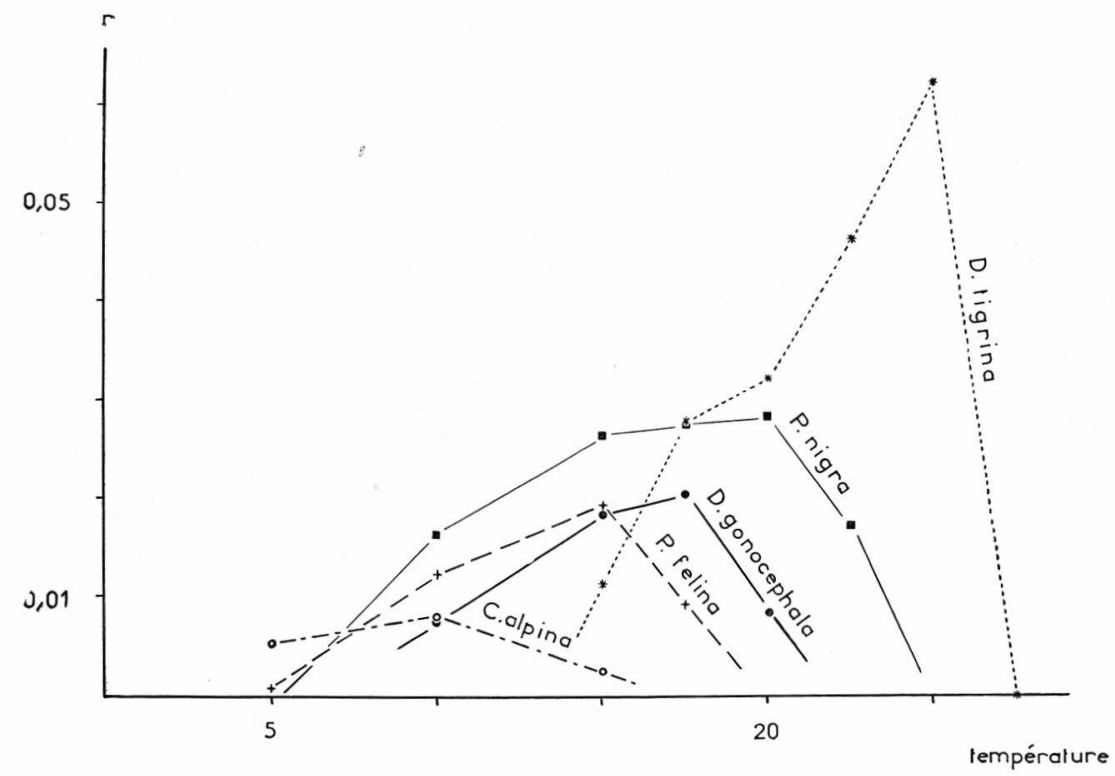

Fig. 3. - Variation du taux intrinsèque d'accroissement $\mathrm{r}$ en fonction de la température chez les Planaires. Les droites de Crenobia alpina, Polycelis felina, Dugesia gonocephala et Polycelis nigra sont transcrites des publications de Pattee 1919,1970 et 1972. 
La courbe correspondant à $D$. tigrina est décalée vers les températures supérieures. Les 5 espèces sont rangées dans un ordre de thermophilie croissante.

Le rendement de $D$. tigrina, ou capacité à transformer les ressources alimentaires en descendants, est supérieure à celui de C. alpina à partir de $15^{\circ}$. Entre $15^{\circ}$ et $17,5^{\circ}$ D. gonocephala, $P$. felina et $P$. nigra sont victorieuses. A $17,5^{\circ} P$. nigra et $D$. tigrina montrent le même rendement.

Dans les conditions de l'expérience, D. tigrina l'emporte, audessus de $20^{\circ}$, sur les 4 espèces indigènes.

Les zones où la reproduction est possible sont à peu près de la même largeur pour chacune. La position de $D$. tigrina par rapport aux autres Planaires, sur cette figure, est en accord avec la situation qu'elle occupe sur le terrain. Tolérance thermique, capacité reproductive et habitat semblent bien liés.

\section{C. - Reproduction à température variable.}

Un lot de $D$. tigrina, comportant 20 adultes répartis dans 2 bols et traités rigoureusement comme les animaux élevés à température constante, a été installé dans une pièce vitrée et non chauffée, ouverte en permanence sur l'extéricur et dont la température varie suivant les saisons. Cette température est mesurée au moyen d'un thermomètre à maxima et minima relevé toutes les semaines.

J'ai mesuré le taux de fragmentation des animaux chaque mois. Ce taux est défini comme le nombre moyen de jeunes produits par un animal pendant la période considérée. J'ai pu ainsi suivre le cycle de reproduction de cette espèce en fonction des températures régnant dans les eaux de notre région.

Les résultats figurent au tableau VI. La température moyenne mensuelle est calculée à partir des températures extrêmes hebdomadaires. La figure 4 représente les variations, en fonction du temps, du taux de fragmentation et de la température moyenne. A partir de mai 1970 sont indiquées les températures extrêmes mensuelles.

A température constante, le déplacement des fragments, lorsqu'il se produit, a lieu toute l'année. Les variations dans la fréquence semblent dues à l'âge des animaux et non à la saison. L'influence de cette dernière, si elle existe, est minime.

Curtis (1902) constatait que, dans la nature, la race asexuée de $D$. tigrina ne se reproduisait pas en hiver, mais sculement de juin à octobre. L'élevage à température variable confirme l'observation de cet auteur et nous montre que la multiplication asexuée de $D$. tigrina suit nettement un cycle saisonnier. Ce cycle est grossièrement parallèle à celui des températures. 


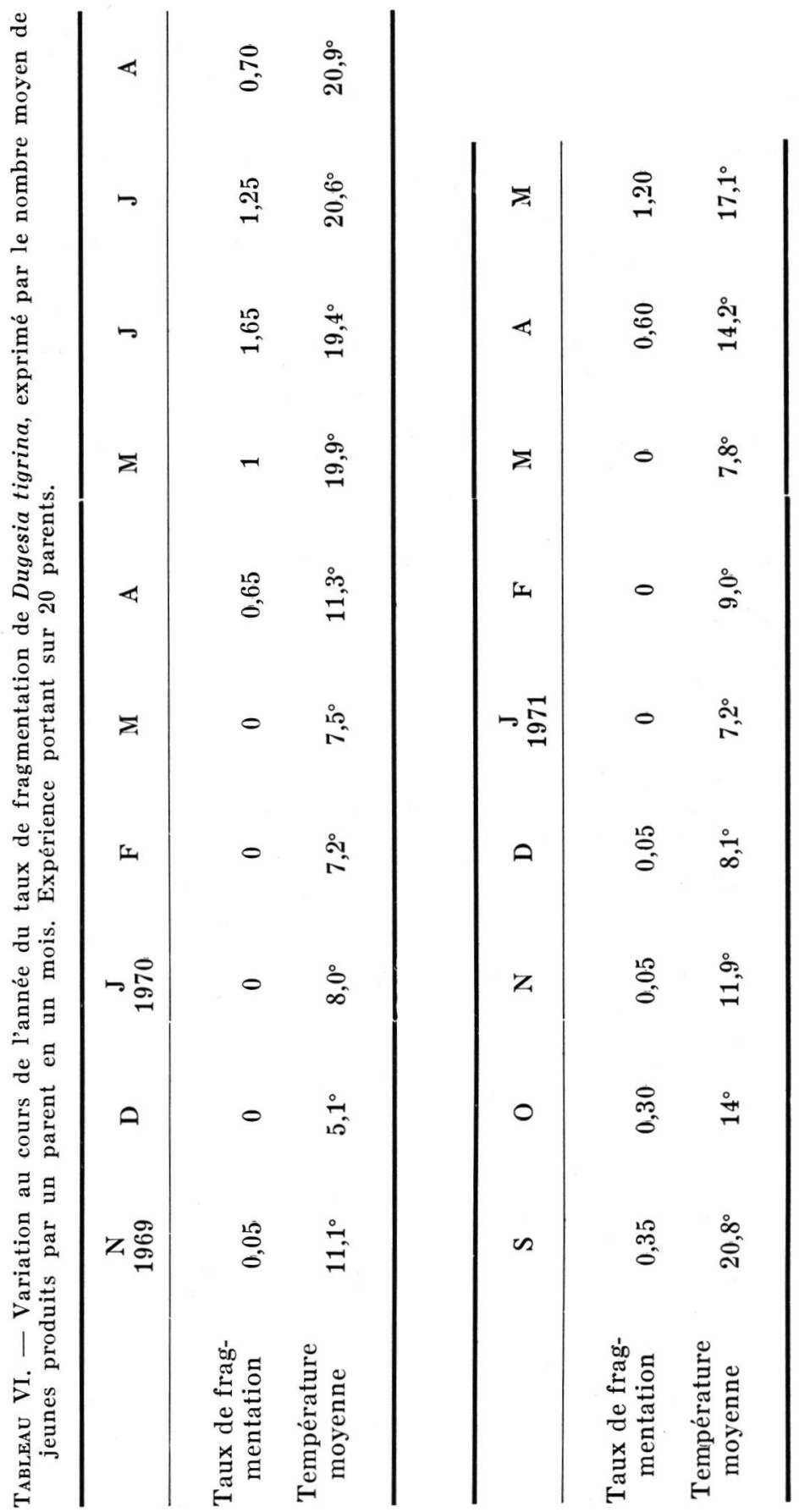




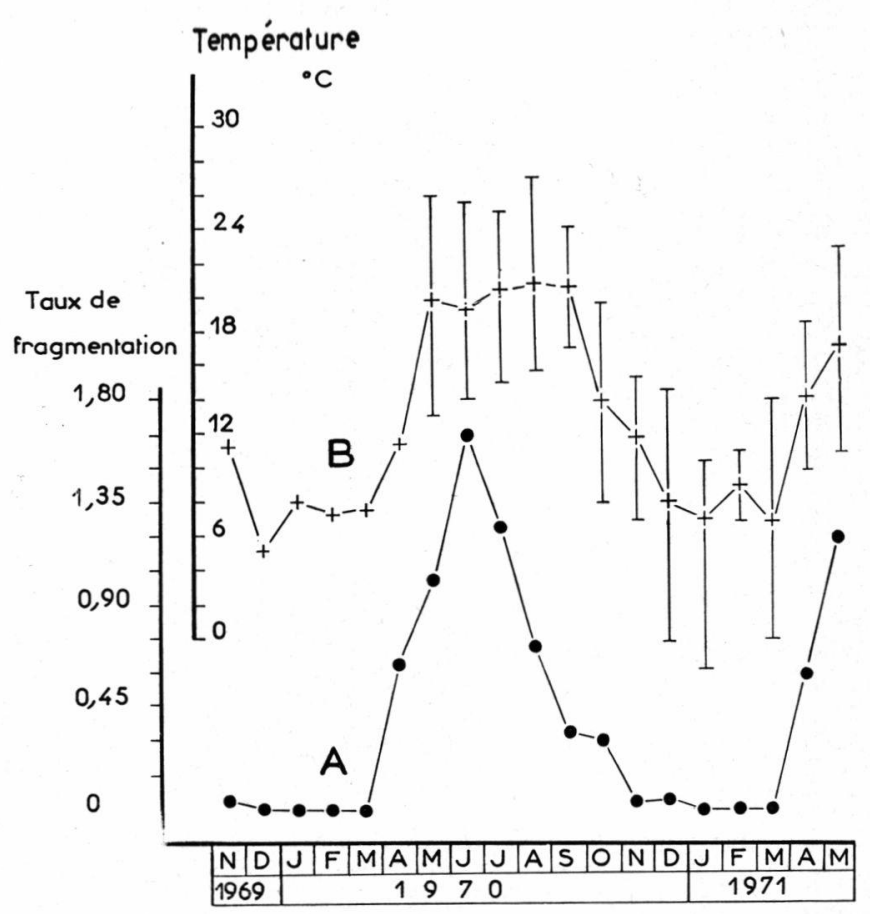

Fig. 4. - Variation au cours de l'année du taux de fragmentation de Dugesia tigrina (courbe A) et de la température (courbe B : moyennes et températures extrêmes mensuelles).

La figure 4 montre cependant que, pour des températures identiques, le taux de fragmentation est différent au printemps et à l'automne, c'est-à-dire selon que le milieu est soumis à un réchauffement ou à un refroidissement progressif. Cette influence primordiale du passé des individus s'explique aisément par les mécanismes qui ont été mis en évidence aux paragraphes précédents.

Nous pouvons distinguer trois phases dans ce cycle annuel de reproduction au laboratoire. Pendant la période froide, de décembre à mars, les individus passent par une phase de croissance : la température est trop basse pour permettre la fragmentation, les dépenses énergétiques sont faibles et la nourriture absorbée permet une augmentation de la taille du corps. Celui-ci peut atteindre des dimensions considérables $(10 \mathrm{~mm}$ environ $)$, constituant une sorte de réserve pour l'animal. En avril, la température moyenne dépassant $10{ }^{\circ} \mathrm{C}$, la multiplication de ces grands individus commence. Ceci correspond bien aux résultats obtenus à température constante. Cette deuxième phase dure jusqu'en juin. A 
chaque nouveau réchauffement correspond une diminution de la taille d'équilibre et donc une tendance supplémentaire à la fragmentation. Vers la fin de cette période, la température a cessé de croitre et les animaux retrouvent progressivement une taille bien plus faible que celle qu'ils avaient au départ, en hiver. Pendant la troisième phase, de juillet à novembre, les fragmentations s'espacent de plus en plus. La température est à son maximum pendant plusieurs mois. Les animaux ont un métabolisme élevé et dépendent entièrement de leur nourriture pour couvrir à la fois leurs besoins énergétiques et assurer la régénération nécessaire à la coupure suivante. A la fin de cette période, la multiplication cesse complètement lorsque, la température décroissant, le seuil constitué par la taille d'équilibre augmente de nouveau.

Il apparaît donc, en définitive, que la variation de la température joue un rôle au moins aussi important que le niveau même de ce facteur.

Cette expérience se rapproche plus que les précédentes des conditions naturelles : sur le terrain, c'est bien en juin que l'on trouve la densité de population la plus élevée et que l'on récolte le plus de $D$. tigrina fragmentées et de petite taille. Mais il faut s'attendre néanmoins à ce que la situation soit compliquée par l'intervention de facteurs autres que la température. L'alimentation, en particulier, est beaucoup plus limitéc. Sa carence doit alors causer un arrêt plus rapide de la multiplication en été, ainsi que l'ont constaté REynoldson $(1960,1964)$ ou Taylor et Reynoldson (1962) pour d'autres espèces de Planaires.

\section{III. - CONCLUSIONS}

Les mesures au laboratoire montrent que, du point de vue de la capacité de survie, $D$. tigrina est la Planaire la plus eurytherme. Elle résiste à des températures comprises entre $5^{\circ} \mathrm{C}$ et $27,5^{\circ} \mathrm{C}$. L'effet létal des températures élevées ne se fait sentir qu'à partir de $32,5^{\circ} \mathrm{C}$, où elle survit un mois environ. Ses rivales les plus fréquentes : $P$. nigra et $P$. tenuis survivent pendant le même temps à $28{ }^{\circ} \mathrm{C}$ pour la première, $29^{\circ} \mathrm{C}$ pour la deuxième (LASCOMBe 1971). Des conditions aussi dures ne règnent pas sous nos climats. Cet effet ne peut donc guère avoir d'influence directe ou indirecte sur la répartition de $D$. tigrina.

Cependant notre Planaire est capable de se reproduire seulement dans une zone de température bien délimitée et qui n'est pas plus large que celle de la plupart des autres espèces (fig. 3). L'animal se révèle seulement plus thermophile que ces dernières, 
et la zone à laquelle il est adapté est décalée vers les températures élevées. Son écophysiologie peut se résumer de la façon suivante :

- Les basses températures ont un rôle limitant seulement par la durée pendant laquelle elles règnent. En effet dans nos régions, en hiver, quand la température varie entre $0{ }^{\circ} \mathrm{C}$ et $5{ }^{\circ} \mathrm{C}, D$. tigrina survit mais est inactive. Ces conditions sont donc défavorables à notre Planaire, en comparaison des espèces indigènes qui s'alimentent, atteignent de grandes dimensions et accumulent des réserves. Certains comme $C$. alpina et $P$. felina se reproduisent même.

- A $10{ }^{\circ} \mathrm{C}$, notre espèce s'alimente. Les animaux ont une taille proche du seuil nécessaire à leur fragmentation, mais le niveau thermique est trop bas pour que celle-ci se produise. Par contre, toutes les espèces indigènes étudiées par Patree se reproduisent à cette température.

- Le réchauffement printanier, entraînant une augmentation de la température au-dessus de $10{ }^{\circ} \mathrm{C}$, coïncide pour $D$. tigrina avec l'utilisation des réserves et le début de la reproduction. A partir de $20^{\circ} \mathrm{C}$, le taux d'accroissement est supérieur à celui des espèces indigènes; il atteint son niveau maximum à $25^{\circ} \mathrm{C}$. Entre $27,5^{\circ} \mathrm{C}$ et $32,5^{\circ} \mathrm{C}$ la température permet la multiplication, mais les effets de cette dernière, ajoutés au métabolisme élevé, entrâ̂nent une diminution continuelle de la taille des individus, qui détachent finalement des fragments non viables car trop petits.

Du point de vue thermique l'implantation de $D$. tigrina dans nos régions est tributaire de plusieurs exigences :

- La première est évidemment que la température dépasse $10{ }^{\circ} \mathrm{C}$ pendant une durée suffisante pour assurer la reproduction de l'espèce. L'absence de celle-ci dans les lacs froids et à l'amont des cours d'eau est à mettre en liaison avec la diminution de son activité générale et de ses capacités reproductives aux basses températures.

- Si l'animal doit entrer en compétition avec les espèces indigènes, ce qui est généralement le cas, son succès dépendra entre autres des deux circonstances suivantes : le temps pendant lequel la température dépassera $18{ }^{\circ} \mathrm{C}$ et atteindra les niveaux où le taux d'accroissement de $D$. tigrina est supérieur à celui de ses rivales et, inversement, le temps pendant lequel cette température se maintiendra dans la zone défavorable aux alentours et en dessous de $5{ }^{\circ} \mathrm{C}$. D . tigrina se montre ainsi adaptée à exploiter la niche écologique située à l'extrémité la plus chaude de la série thermique occupée par les Planaires existant actuellement dans la région. 


\section{RÉSUMÉ}

La capacité reproductrice de $D$. tigrina est mesurée à différents niveaux de température. Elle est exprimée par le taux intrinsèque d'accroissement naturel. La reproduction, inhibée par le froid jusqu'à une température légèrement supérieure à $10^{\circ}$, commence dès que ce seuil est dépassé et cesse au-dessus de $25^{\circ}$. Le taux d'accroissement est supérieur à celui des espèces indigènes à partir de $20^{\circ}$.

A chaque température, les individus ont tendance à atteindre une taille d'équilibre. Une relation est mise en évidence entre cette dernière et l'intensité de la reproduction. Au-dessous de la taille d'équilibre les animaux présentent une phase de croissance, au-dessus la multiplication commence éventuellement. Ce phénomène est confirmé à des températures qui varient en suivant le cycle extérieur. Dans ce cas, la fragmentation est saisonnière et présente un maximum en juin.

\section{THE TEMPERATURE RELATIONS OF SOME FRESH-WATER PLANARIANS AND THEIR INCIDENCE IN ECOLOGY}

\section{6. -- DUGESIA TIGRINA}

The reproductive capacity of $D$. tigrina was determined at different levels of temperature. It was measured by the intrinsic rate of natural increase of the population. Reproduction was inhibited by cold up to a little more than $10^{\circ}$. It began above this level and ceased again from about $25^{\circ}$ upwards. The rate of increase of $D$. ligrina was found to be greater than that of the local species at $20^{\circ}$ and above.

At each temperature level, the animals tend to reach a characteristic size of equilibrium. A relation between this size and the occurence of reproduction was demonstrated. Animals smaller than this characteristic size tend to grow, animals larger than this size eventually multiply. These processes were found to be also valid in the case of temperature fluctuating according to the outdoor cycle. Under these conditions, scissions occured seasonally and were at their highest in june.

\section{TRAVAUX CITÉS}

Аввотт (В. J.). 1960. - A note on the oxygens and temperature tolerances of the Triclads Phagocata gracilis (Haldeman) and Dugesia tigrina (Girard). Va. J. Sci. n.s., $11: 1-8$.

Abeloos (M.). 1929. - Influence de la température sur la croissance des Planaires. C.R. Acad. Sci., Paris, $188: 881$.

Abeioos (M.). 1930. - Recherches expérimentales sur la croissance et la régénération chez les Planaires. Bull. biol. Fr. Belg., 64 : 1-140.

Chandeer (C. M.). 1965. - Environmental factors affecting the local distribution and abundance of four species of stream-dwelling Triclads. Indiana Univ., Ph. D. thesis, 1-104.

Cols (L. C.) . 1960. - A note on population parameters in cases of complex reproduction. Ecology, 41 : 372-375.

Cuntis (W. C.). 1902. - The life history, the normal fission and the reproductive organs of Planaria maculata. Proc. Boston Soc. nat. Hist., 30 : 515-559. 
DАнм (A. G.). 1955. - Dugesia tigrina (Girard) an American immigrant into European waters. Verh. int. Ver. Limnol., 12 : 554-561.

DAHM (A. G.). 1958. - Taxonomy and ecology of five species groups in the family Planariidae (Turbellaria Tricladida Paludicola). Malmö, 241 p.

Fry (F. E. J.), Hart (J. S.) et Walker (K. F.). 1946. - Lethal temperature relations for a sample of young speckled trout: (Salvelinus fontinalis). Univ. Toronto Stud. biol. Ser., 54. Publs. Ont. Fish. Res. Lab., 66 : 5-35.

KeNK (R.). 1937. - Sexual and asexual reproduction in Euplanaria tigrina (Girard). Biol. Bull. mar. biol. Lab., Woods Hole, 73 : 280294.

Lascombe (C.). 1971. - Recherches écologiques et biogéographiques sur deux espèces jumelles de Planaires d'eau douce dans la région lyonnaise. Thèse, Lyon, $116 \mathrm{p}$.

Lison (L.). 1958. — Statistique appliquée à la biologie expérimentale. Paris, 346 p.

Mathieu (J.). 1971. - Influence de la durée d'acclimatation thermique sur la durée de survie aux hautes températures, chez Niphargus longicaudatus (Amphipode, Gammaridé des eaux souterraines). Naturaliste can., $98: 59-68$.

Pattee (E.). 1966. - Coefficients thermiques et écologie de quelques Planaires d'eau douce. 1. Tolérance des adultes. Annls Limnol., $2: 469-475$.

Pattee (E.). 1968. - Coefficients thermiques et écologie de quelques Planaires d'eau douce. 2. Tolérance de Dugesia gonocephala. Annls Limnol., 4 : 99-104.

Patree (E.). 1969. - Coefficients thermiques et écologie de quelques Planaires d'eau douce. 3 . La reproduction des deux espèces montagnardes. Annls Limnol., 5 : 9-24.

Pattee (E.). 1970. - Coefficients thermiques et écologie de quelques Planaires d'eau douce. 4. La reproduction de Dugesia gonocephala. Annls Limnol., 6 : 293-304.

Pattee (E.). 1972. - Coefficients thermiques et écologie de quelques Planaires d'eau douce. 5. La reproduction des deux espèces limnophiles Polycelis nigra et Polycelis tenuis. Annls Limnol., 8 :

Rexnoldson (T. B.). 1960. - A quantitative study of the population biology of Polycelis tenuis (Ijima) (Turbellaria, Tricladida). Ö̈kos, 11 : 125-141.

Reynoldson (T. B.). 1964. - Evidence for intra-specific competition in field populations of Triclads. J. Anim. Ecol., 33 : 187-201.

Root (R. B.). 1960. - An estimate of the intrinsic rate of natural increase in the Planarian, Dugesia tigrina. Ecology, 41 : 369-372.

Russier (R.) et Lascombe (C.). 1970. — La Planaire américaine Dugesia tigrina dans la région lyonnaise : écologie et tolérance thermique. Bull. mens. Soc. linn. Lyon, $39: 197-206$.

TAYlor (M. C.) et ReYnoldson (T. B.). 1962. - The population biology of lake-dwelling Polycelis species with special reference to $P$. nigra (Müll.) (Turbellaria, Tricladida). J. Anim. Ecol., 31 : 273-291.

VANDEL (A.). 1922. - Recherches expérimentales sur les modes de reproduction des Planaires Triclades Paludicoles. Bull. biol. Fr. Belg., $55: 343-518$. 